\title{
The marriage problem: from the bar of appointments to the agency
}

\author{
Alejandro Lage-Castellanos and Roberto Mulet \\ Henri-Poincaré Group of Complex Systems, Physics Faculty, \\ University of Havana, La Habana, CP 10400, Cuba and \\ Department of Theoretical Physics, Physics Faculty, \\ University of Havana, La Habana, CP 10400, Cuba
}

(Dated: August 13, 2018)

\begin{abstract}
We study the stable marriage problem from different points of view. We proposed a microscopic dynamic that lead the system to a stationary state that we are able to characterize analytically. Then, we derive a thermodynamical description of the Nash equilibrium states of the system that agree very well with the results of Monte Carlo simulations. Finally, through large scale numerical simulations we compare the Global Optimum of the society with the stable marriage of lower energy. We showed that both states are strongly correlated and that the selffish attitude results in a benefit for most of the practitioners belonging to blocking pairs in the Global Optimum of the society.
\end{abstract}

PACS numbers: 05.20.-y, 01.75.+m, 02.50.Le

\section{INTRODUCTION}

The Stable Marriage Problem (SMP) [1] describes a system where two classes of agents (e.g. men and women) have to be matched pairwise. To each player is assigned a list of preferred partners and the aim of the problem is to find those states that are stable with respect to single agent decisions, i.e. those states that are Nash equilibria [2].

A Nash equilibrium state is a state in which, being the strategies of the other players constant, any variation of an agent's strategy results in a worse performance for him The concept has become a fruitful source of inspiration for physicists, and many "games" have been studied in this context. At variance with usual optimization problems, where the task is to maximize a global function, in Game Theory the main goal is to maximize the utility of the agents of the system. In this context, the marriage problem has a parallel optimization analogous, the Bipartite Weighted Matching Problem (BWMP) [3] , that is also usually known as the Assignment Problem. In the language of the marriage the BWMP consists in the determination of the state that maximizes the global happiness of the society, or alternatively the state that minimizes the unhappiness.

Putting it in other words, given two sets of $N$ agents, each one with a preference list for the possible partners, two kind of problems are well posed: a) to find the assignment that maximizes the happiness of the society (BWMP), b) to find those assignments which are stable with respect to the individual decision of the agents (SMP).

From the technical point of view, both problems are described assigning to each man (woman) an energy $x=X / N$ $(y=Y / n)$ if he(she) is married with a woman(man) ranked $X(Y)$ in their list of preferences. The energy of the system is therefore calculated as:

$$
E=\sum_{i=1}^{N} x_{i}+\sum_{j=1}^{N} y_{j}
$$

and the BWMP problem is reduced to the search of the assignment $\Pi$ that minimizes $E$ while the Stable Marriage Problem to the search of those states that are Nash equilibria.

Both problems have algorithmic solutions in polynomial time, and the general properties of these solutions have been well studied in recent years [4, 5]. Moreover, the marriage problem has being extended to more realistic situations, usually imposing limited information to the agents [] , or assuming correlations in the list of preferences []]. However many relevant questions are still open: How does a real system reach the Nash states? How stable is a Nash state to external perturbations? Or how much similar are the Optimum Global state of the system and a Nash state? This last question for example, may gives some hint about the tendency of the agents of a system, optimally matched, to act for self benefit.

From the algorithmic point of view the assignment problem is usually assumed as a model where a matchmaker decides who to pair with to optimize the happiness of the society. In contrast, the solution of the Marriage Problem is considered as the natural stable state to which the society evolves assuming infinitely rational agents that share all the information of the game. However, it must be keep in mind that the reality is by far more complex. The dynamics of interaction between men and women, employers and employees, buyers and sellers, etc, in society and economy is only rarely enclosed in these kind of algorithms. Usually the dynamics is more rich and do not warranty a convergence to a Nash equilibrium. 
Therefore our aim in this paper is threefold, first to introduce and to study a local microscopic dynamics for the marriage problem that leads to a stationary state, (not necessary a Nash state). Second, to study the evolutionary dynamics, between Nash states, of the marriage problem and to enclose it in a convenient thermodynamical formalism. And finally to study the transition from the Global Optimum state, decided by a matrimonial agency (matchmaker) to a Nash state and vice-versa.

The remaining of the paper is organized as follows. In the next section we introduce a microscopic dynamic for the marriage problem and propose a mean field description of this dynamic that characterizes very well the stationary state of the system. From this solution we are able to derive a strategy to improve the distribution of the happiness in the society. Then, in section III we propose a thermodynamical description of the stationary states of the marriage problem. We compare the analytical and numerical results with computer simulations and show a perfect agreement. Then, in section [V] we compare the statistical properties of the best Nash state with the global optimum of the system. Finally, the conclusions are outlined.

\section{THE BAR OF APPOINTMENTS}

In this section we introduce a new dynamics to model the evolution of two kind of agents in the society. Keeping the analogy of the marriage problem, we consider $N$ men and $N$ women, each one with a list of preferences. At $t=0$ the system is prepared in a random configuration. Then, a man, belonging to a couple (let us call it, couple $A_{o}$ ), and a woman from a different couple, $\left(B_{o}\right)$ go to a bar (the bar of appointments) and meet together. Then, if both prefer to stay together, than with their original couples, (i.e. they are better ranking in their respective preference list than the current spouse and husband), they form a new couple $A_{f}$. Then, the abandoned partners, because of the lonely form a new couple too $B_{f}$. The process is repeated and at each time step, a man and a woman from different couples meet at the bar and decide what to do.

Compared with the usual Marriage Problem this model may be considered a limit situation where the preference lists of the players are hidden to all, but the owner, a situation also closer to the reality. The model tries to be a simple cartoon of the dynamical behavior of many economical and social systems. For example, in a completely free market job, a worker have a meeting with a company. If the company sees that the new worker may be more useful than some other already employed, and at the same time the worker realizes that the offer of the company improves his current position, then, he will choose to resign and the company to assume him substituting one of his employees. The fired employee, will go to the company of the former agent, now with a vacant position, and will be immediately assumed.

One may wonder if this kind of dynamics may lead to a Nash equilibrium situation. Note that, being this the case, the system will be blocked forever. In fact, the system will be in Nash equilibrium, if and only if, there are not men and women that prefer to stay together than with their actual partners. Therefore, the Nash state are stable points of this dynamic.

In figure 1 we plot the probability density $\rho(x)$ obtained from the simulations that in the steady state a man(woman) has energy $x(y)$.

To analytically study this stationary state, we develop a mean field description of this dynamic. At each time step, the man (woman) that go to the bar, meet a woman (man) that is randomly located in his (her) preference list and this location is independent of the person met. In other words, at variance with the model described above where the preference lists are fixed at the beginning of the simulation, now the lists change every time that the agents go out from the bar.

Under this new assumptions, we are able to determine which is the probability $\rho(x, y)$ to find a couple where a man and a woman have energies $x$ and $y$ respectively. As usual, a man has energy $x=X / N$ if he is married with the woman ranked $X$ in his list of preferences, and similarly a woman has energy $y=Y / N$ if she is married with a man ranked $Y$ in her list. The master equation of the stochastic process described above may be written:

$$
\frac{\partial \rho(x, y)}{\partial t}=\int_{(a, b)=0}^{1}\left(P_{(a, b) \rightarrow(x, y)} \rho(a, b)-\left(P_{(x, y) \rightarrow(a, b)} \rho(x, y)\right) d a d b\right.
$$

where $P_{(a, b) \rightarrow(x, y)}$ is the probability per unit time that a man who belongs to a couple with energies $(a, b)$ goes to a new couple with energies $(x, y)$ after an appointment in the bar.

The first couple may be involved in a succeful appointment in two ways, either the man of the couple meets a new woman preferred to the one he is married with, or the woman meets a new man preferred to her current husband. 


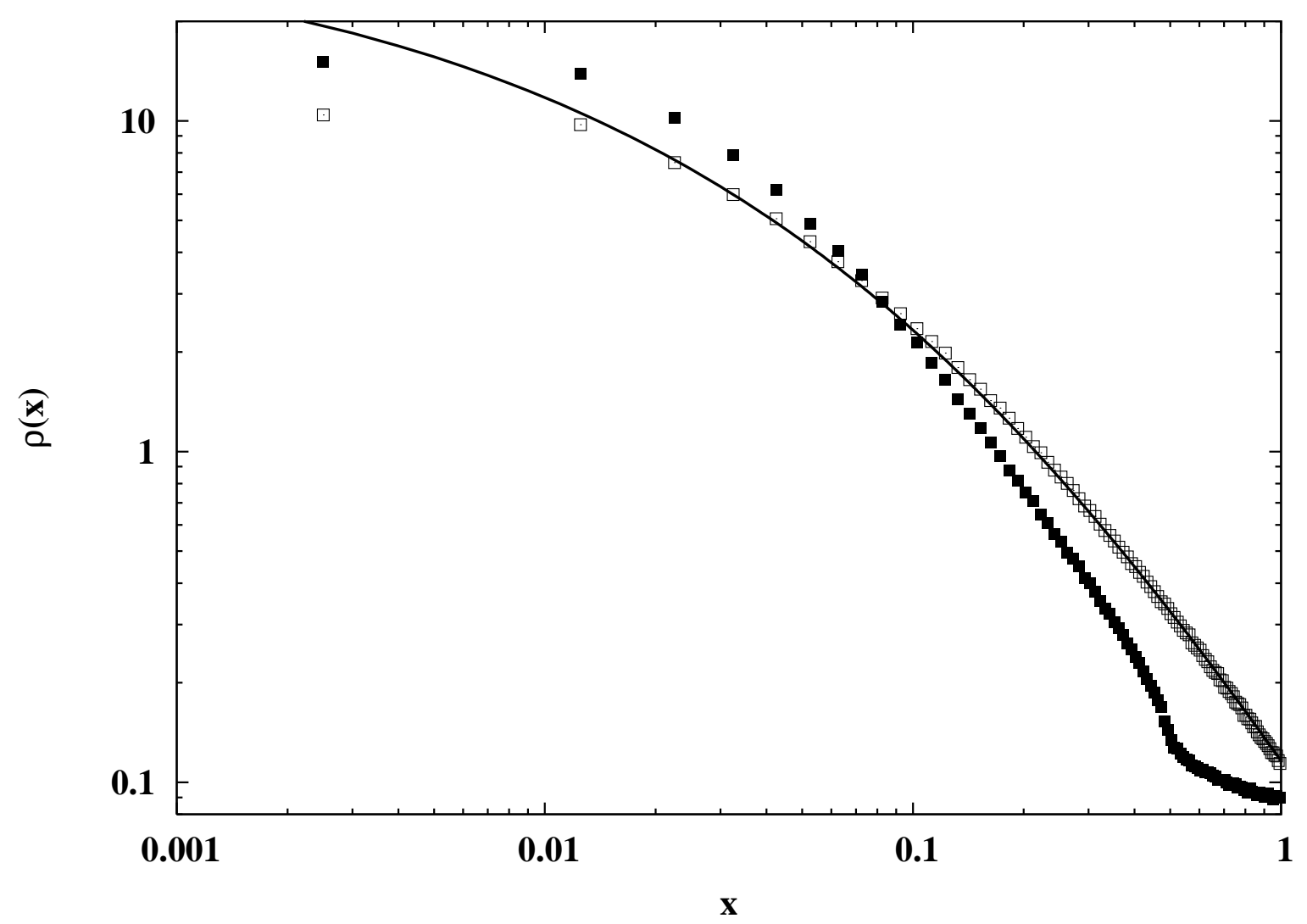

FIG. 1: Stationary energy distribution for the bar of appointments (open symbols) and the associated smarter strategy $N=500$ (close symbols). Symbols indentify simulation results for $N=500$ averaged over 1000 instances. The continuos line represent the analytical solution of the model.

Keeping track of the different ways in which this may happen (see appendix (VI), the equation (2) may be written as:

$$
\frac{\partial \rho(x, y)}{\partial t}=\int_{0}^{1} \int_{x}^{1} \rho(a, b) d a d b \int_{0}^{1} \int_{y}^{1} \rho(a, b) d a d b+<x>^{2}-<x>\rho(x, y)(x+y)
$$

where $\langle x\rangle$ is the mean energy of the men, and we used the fact that because of the symmetry of the problem $<x>=\langle y>$.

To study the stationary solution of the problem, we make the right hand side of (3) equal to zero and obtain a closed, non-linear integral equation for $\rho(x, y)$.

$$
\rho(x, y)=\frac{\int_{0}^{1} \int_{x}^{1} \rho(a, b) d a d b \int_{0}^{1} \int_{y}^{1} \rho(a, b) d a d b}{<x>(x+y)}
$$

Then, defining:

$$
F(x)=\int_{0}^{1} \int_{x}^{1} \rho(a, b) d a d b
$$

the probability density of having a couple with energies $(x, y)$ becomes $\rho(x, y)=\frac{F(x) F(y)+<x\rangle^{2}}{\langle x\rangle(x+y)}$ and substituting it in equation (5) we get:

$$
F(x)=\int_{0}^{1} \int_{x}^{1} \frac{F(x) F(y)+<x>^{2}}{<x>(a+b)}
$$


which can be further simplified for numerical porpoises using another auxiliary function:

$$
T(a)=\int_{0}^{1} d b \frac{1}{<x>} \frac{F(b)}{a+b}
$$

such that:

$$
F(x)=\int_{x}^{1} \operatorname{daT}(a) F(a)-(x \log (1+1 / x)+\log (1+x)-\log 4) \frac{1}{x}
$$

In this way, we obtain two coupled equations, one for $T(x)$ (77) and another for $F(x)$ (8) that can be solved in linear time. From $F(x)$ we can trace back $\rho(x, y)$, and integrating with respect to one of the variables, $\rho(x)=\int_{0}^{1} \rho(x, y) d y$ the distribution of the energies of the men (or women) in the stationary state.

The solution for $\rho(x)$ is presented with a continuous line in figure 1 The perfect coincidence with the simulations, shows not only that the system is unable to reach a Nash equilibrium, but also that despite of their existence, and the fact that they are stable states of the simulation, they do not affect the dynamic proposed.

From the exact solution, we may obtain other properties of the distribution, for example, the mean energy of the men.

$$
X=N<x>=N \int_{0}^{1} x \rho(x) d x \approx 0.167 N
$$

in excellent agreement with the simulation.

This result should be also compared with the solution of the problem obtained by optimization algorithms. While with the dynamics proposed above, the energy growths linearly with $N$ (9) in the original assignment and marriage problems the energies growth as $N^{1 / 2}$. This impose, of course not only a quantitative difference in the type of states reached, Nash equilibria for the marriage, global equilibrium for the assignment, and a global stationary state for our dynamics, but also a qualitative difference between the previous two approaches and our dynamics. This may suggest that a real society, where the information of the agents is very limited, has less capabilities for optimization than previously thought by the analysis of the former problems.

Another conclusion that may be easily drawn from the distribution of energies of the couples concerns the balance of energies between its members. Writing $\rho(x, y)$ as a function of $\xi=x+y$, the energy of the couple, and $\delta=x-y$, the energetic unbalance of the couple, it is evident that $\rho(\xi, \delta)$ will be pair with respect to $\delta$ and therefore it will have an extreme at $\delta=0$.

By numerical inspection it is shown (see figure 2) that for small values of the energies $\rho(\chi, \delta)$ has a minimum. This means that couples with small energies (i.e. optimized), are very unbalanced, if one of the members is very happy, the other is unsatisfied. This is the price that the agents pay for acting in self-interest. They will spend half of their life being the happiest of the relation, sharing with a partner that they appreciate, while the other half of their life they will be very unsatisfied.

Following the previous reasoning, we may imagine players that adopt a smarter strategy when visiting the bar. They just need to have a little more information. A man(woman), needs to know not only his preference list but the one of the woman(men) he or she encounters in the bar. Now, a man visiting the bar, will choose a new couple, not only because she is better ranked that his actual partner, but because she may guarantee a long term satisfaction.

Let us, for simplicity, describe first this strategy for a man. Imagine a man with energy $x$, in average if his wife has energy $y$ he will be abandoned after a time $1 / y$. Once he is abandoned he will next marry with a woman with random energy, in average 0.5. Therefore, if he has to decide between two women $w$ and $w^{\prime}$ with energies $y>y^{\prime}$ respectively he follows the next reasoning: If I marry the woman $w^{\prime}$ I will be with her a time $1 / y^{\prime}$ being unhappy a quantity $x^{\prime}$, while to marry the woman $w$ will make me unhappy a quantity $x$ for a time $1 / y$ and a quantity 0.5 for a time $1 / y^{\prime}-1 / y$. Then, a man will choose the woman $w^{\prime}$ only if she minimizes his unhappiness during this time, i.e. if:

$$
\frac{x^{\prime}}{y^{\prime}}<\frac{x}{y}+0.5\left(\frac{1}{y^{\prime}}-\frac{1}{y}\right)
$$

or, equivalently:

$$
\frac{x^{\prime}-0.5}{y^{\prime}}<\frac{x-0.5}{y}
$$




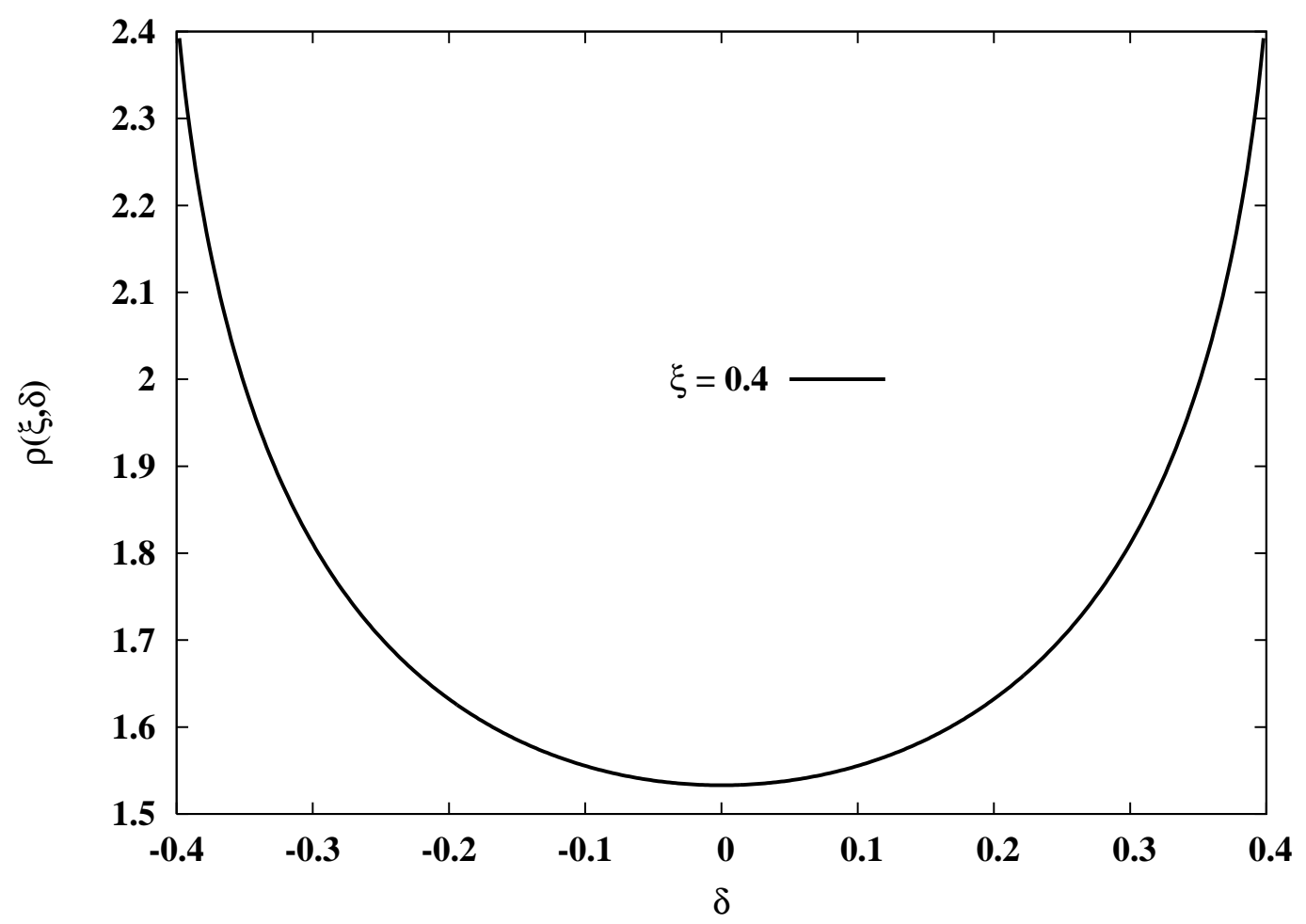

FIG. 2: Probability to find a copy with energy $\xi$ as the function of the unbalance $\delta$

Since the equation (11) is symmetric with respect to $w$ and $w^{\prime}$ the man will choose the woman that minimizes $\frac{x-0.5}{y}$. A woman, of course will behave in the same way.

Therefore when a man and a woman meet in a bar, a man will leave her wife only if the value of $\frac{x-0.5}{y}$ with the woman found at the bar is lower than the same fraction calculated with his actual wife. A woman will do the same, but substituting $x$ by $y$ in equation (11).

The results of the simulations using this strategy appear in figure 1 with black symbols. As can be seen this new strategy results in a general improvement of the energies of the agents. The new distribution is higher for low values of $x$ and smaller for large values of $x$ than the previous one. The clear change in the slope of the distribution for high energies shown in the figure suggests that the probability to find a man or a woman with energy close to one is very low.

Calculating the mean energy of the men (or women) we find that it is $6 \%$ lower than the energy estimated with the first strategy. It must also be noted that this improvement was obtained without the necessity to increased considerably the capacity of the agents to get or to process the information and at the same time keeping their self-interest.

From the market labor place, this suggest that a good strategy for a job seeker may be not to look only for the must profitable job, but also for a position where he is useful, such that, in the short run, the employer may find difficult to find a good substitute for him.

\section{THERMODYNAMICS OF THE STABLE STATES}

In this section, we propose a thermodynamical formalism to characterize the behavior of the system, assuming that it moves dynamically between the different Nash equilibria of the game. Compared with the previous model, now, the agents, share the full information of the system and are able to select their best strategy and to reach a Nash equilibrium state.

At $t=0$, men and women will be in a Nash equilibrium state, and some perturbation, that we will associate later with a temperature, kick out the system from this state. However, since the agents are infinitely rational, they immediately rearrange in a new Nash equilibrium state, not necessary the previous one.

A simple dynamics of this kind of behavior can be studied through standard Monte Carlo techniques. Given the 
agents and their preference lists, we first determine the different Nash equilibrium states of the system. Then at $t=0$ we select one at random, and through a Markov process we visit all the stable states, with the following probability, $p_{o \rightarrow f}=\min \left[1, \exp \left(-\beta\left(E_{f}-E_{o}\right)\right)\right]$, where $E_{f}$ and $E_{o}$ are the energies of the final and initial states of the system.

The temperature of the system $1 / \beta$, represents the external drive received by the agents to change their equilibrium state. A low value of $\beta$ reflects a situation where the agents, despite the fact that they are in a Nash equilibrium, tend to change frequently their partners, looking for better matchings. A large value of $\beta$ reflects the opposite, the tendency of the agents to explore new possibilities is strongly reduce.

We calculate the total energy of the system, and its fluctuations as a function of $T$. The results of the simulations appear with symbols in figure 3 and 4 Note that both the total energy and the fluctuations of the system decrease with the temperature. For large temperatures, however, the energy saturates, a finite size effect that will be discussed below.

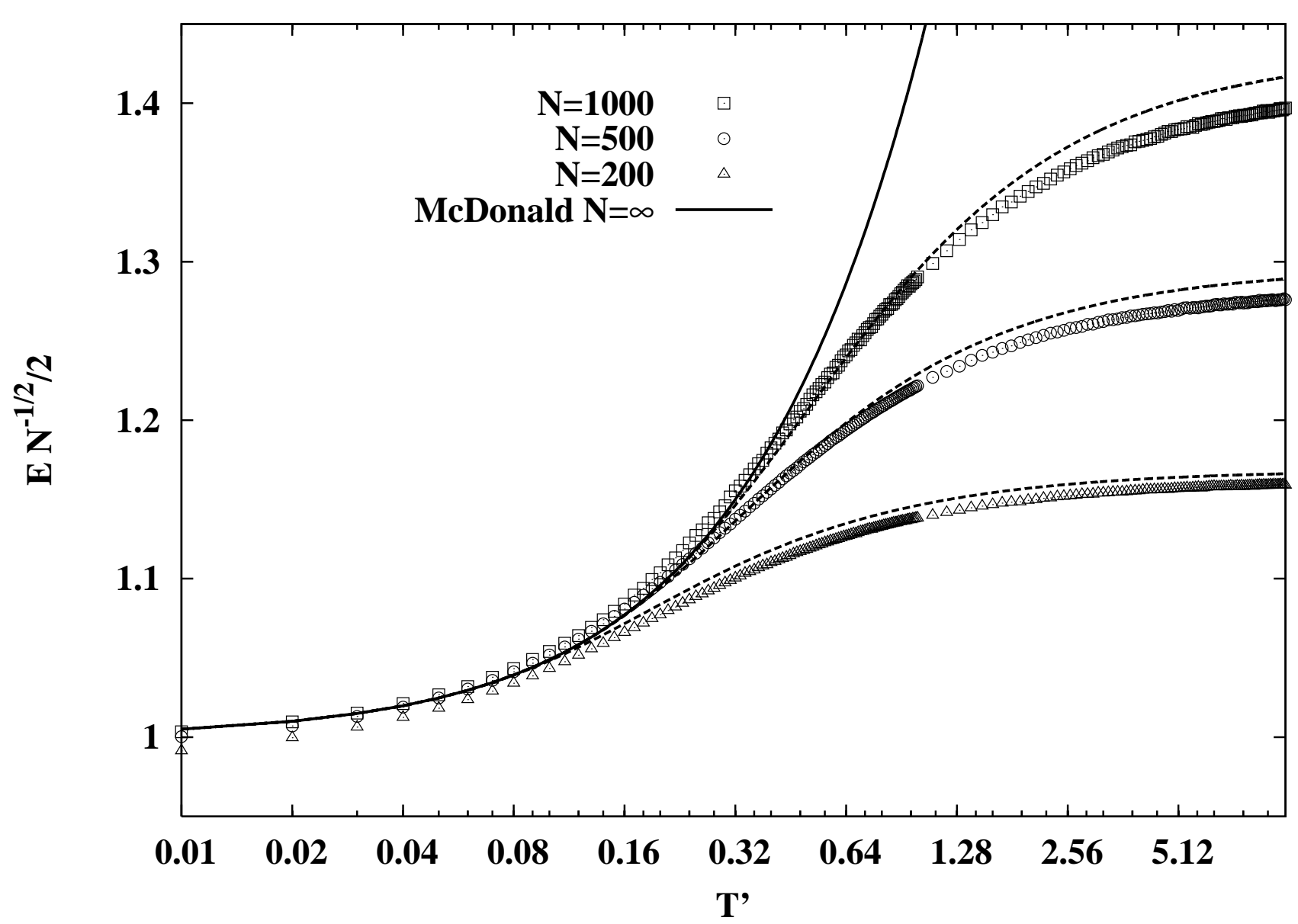

FIG. 3: Energy of the system as a function of the temperature T. Every point is the average over 100 instances during the simulation. The continuous line is the analytical result and the discontinuous one, represents the numerical calculations

To explain these results, we perform a thermodynamical analysis based on the approach developed by Dzierzawa et al $[\underline{5}$. to study the number of equilibrium states of the system.

The number of stable (Nash) states in the system may be written as:

$$
S=\int d X \int d Y \rho(X) \rho(Y) e^{-X Y}
$$

where $X=\sum_{i=1}^{N} x_{i}, Y=\sum_{i=1}^{N} y_{i}$, and $\rho(X)=\frac{X^{N-1}}{\Gamma(N)}\left(1-\exp \left(-\frac{X}{N}\right)\right)^{N}$.

From equation (12) and, the known result that the density of states for $N$ large enough is concentrated around the curve $X Y=N[4]$ we may calculate the density of states of the system: 


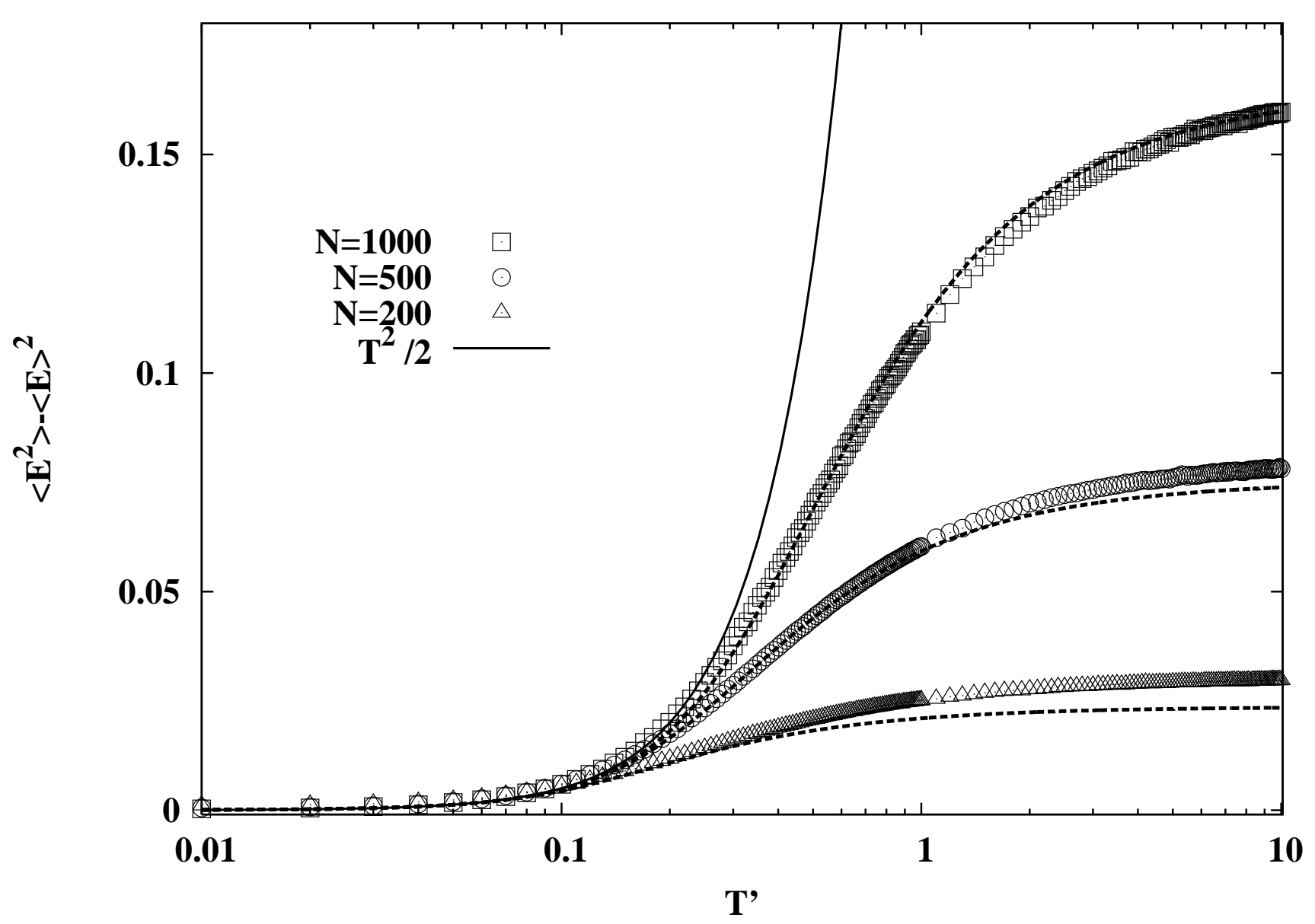

FIG. 4: Specific heat of the system as a function of the temperature T. Every point is the average over 100 instances during the simulation. The continuous line is the analytical result and the discontinuous one, represents the numerical calculations

$$
\begin{aligned}
D(E) & =\int_{0}^{N} d X \int_{0}^{N} d Y \rho(X) \rho(Y) e^{-X Y} \delta(X Y-N) \delta(X+Y-E) \\
& \approx \frac{1}{\sqrt{E^{2}-4 N}}
\end{aligned}
$$

valid in the interval $(2 \sqrt{N}, \log N+N / \log N)$. Then, to get some insight on the thermodynamical behavior of the system we calculate, using (13), the partition function of the system at a finite value of $\beta$ :

$$
\begin{aligned}
Z(\beta) & =\int_{2 \sqrt{N}}^{N / \log N} d E D(E) e^{-\beta E} \\
& =\int_{2 \sqrt{N}}^{N / \log N} d E \frac{e^{-\beta E}}{\sqrt{E^{2}-4 N}}
\end{aligned}
$$

which in the new variable $E^{\prime}=\frac{E}{2 \sqrt{N}}$ may be written as:

$$
Z(\beta)=\int_{1}^{\sqrt{N} /(2 \log N)} d E^{\prime} \frac{e^{-2 \sqrt{N} \beta E^{\prime}}}{\sqrt{E^{\prime 2}-1}}
$$

that can, in turn, be analytically solved in the thermodynamic limit $N \rightarrow \infty$, 


$$
\begin{aligned}
Z(\beta) & \simeq \int_{1}^{\infty} d E^{\prime} \frac{e^{-2 \sqrt{N} \beta E^{\prime}}}{\sqrt{E^{\prime 2}-1}} \\
& =K_{0}(2 \sqrt{N} \beta)
\end{aligned}
$$

where $K_{0}(x)$ is the MacDonald function of order zero. From (16) it is an easy exercise to derivate the thermodynamical variables of the system. The energy follows $[8]$ :

$$
E^{\prime}\left(\beta^{\prime}\right)=\frac{K\left(1, \beta^{\prime}\right)}{K\left(0, \beta^{\prime}\right)}
$$

and the specific heat $[8]$ :

$$
C^{\prime}\left(\beta^{\prime}=1 / T^{\prime}\right)=\beta^{\prime 2}\left(\frac{1}{2}+\frac{K\left(2, \beta^{\prime}\right) K\left(0, \beta^{\prime}\right)-2 K\left(1, \beta^{\prime}\right)^{2}}{2 K\left(0, \beta^{\prime}\right)^{2}}\right)
$$

where $\beta^{\prime}=2 \sqrt{N} \beta$.

These results (equations (17) and (18) appear as solid lines in figures 3 and 4 Note that the agreement with the simulation is very good at low temperatures, but a clear discrepancy exists for large values of $T$.

This discrepancy is due to the approximation used for the calculation of $D(E)$ first, and latter of $Z(\beta)$. In the calculation of the density of states we use the fact that $X Y=N$, a result that is true only for very large $N$. In fact, the comparison of these analytical results (solid line) with the numerical computation (symbols) of the density of states shown in figure [5 reflects that for large values of $E$ this approximation is not longer true. Moreover, taking the thermodynamic limit in equation (15) to get the analytical solution (16) for $Z(\beta)$, is not mathematically justified for $\beta \rightarrow 0$.

A more precise, while not analytical treatment of the problem may be obtained solving numerically, for fixed values of $N$ the equation (15) and the thermodynamical derived equations for $E$ and $C_{v}$. These numerical results appear also in figures 3 and 4 with dashed lines, showing a much better coincidence with the simulations.

Therefore, the comparison of the analytical results obtained above with the Monte Carlo simulations, show that the approach developed by Dzierzawa et al. [5] can be extended to describe the dynamical behavior of these kind of systems. Moreover, the analysis of the results lead us to conclude that a too dynamical society (high temperature) will lead the agents to explore configurations that, while stable are, in average for the society, less satisfactory.

\section{STABLE STATES VERSUS GLOBAL OPTIMIZATION}

It is also worth to compare which are the similarities and differences between the stable states of the marriage problem and the global optimum of the society. The comparison is helpful first because these problems have been rarely studied together beyond the average energy analysis, and to have a more general idea about which are really the differences and similarities of both kind of solutions may give some insight on the interplay between global and personal optimization. Moreover, it can give also some clue on which is the best strategy that should follow a matchmaker (or a matrimonial agency) when facing the problem of pairing two subsets, that can a posteriori take their own decisions.

To make this comparison we performed extensive numerical simulations and compare the statistical properties of the matching in two states, the Global Optimum of the system $\Pi_{0}$, calculated using the Hungarian method [3] , and the Stable Marriage with lower energy $\Pi_{0}^{s}$.

It was already proved that the energy of both kind of problems is different. For the BWMP $E_{B}=1.617 \sqrt{N}[3$, [4] and for the best marriage $E_{M}=2 \sqrt{N}[4]$. Moreover from figure [5 it becomes clear that there is an energetic gap, between the solution of the Marriage Problem and the solution of the Weighted Assignment Problem.

An interesting quantity to measure between both states is the distance, defined as the number of different couples in the two matchings:

$$
D\left(\Pi^{a}, \Pi^{b}\right)=\frac{1}{N} \sum_{i=1}^{N}\left(1-\delta_{\Pi_{i}^{a}, \Pi_{i}^{b}}\right)
$$




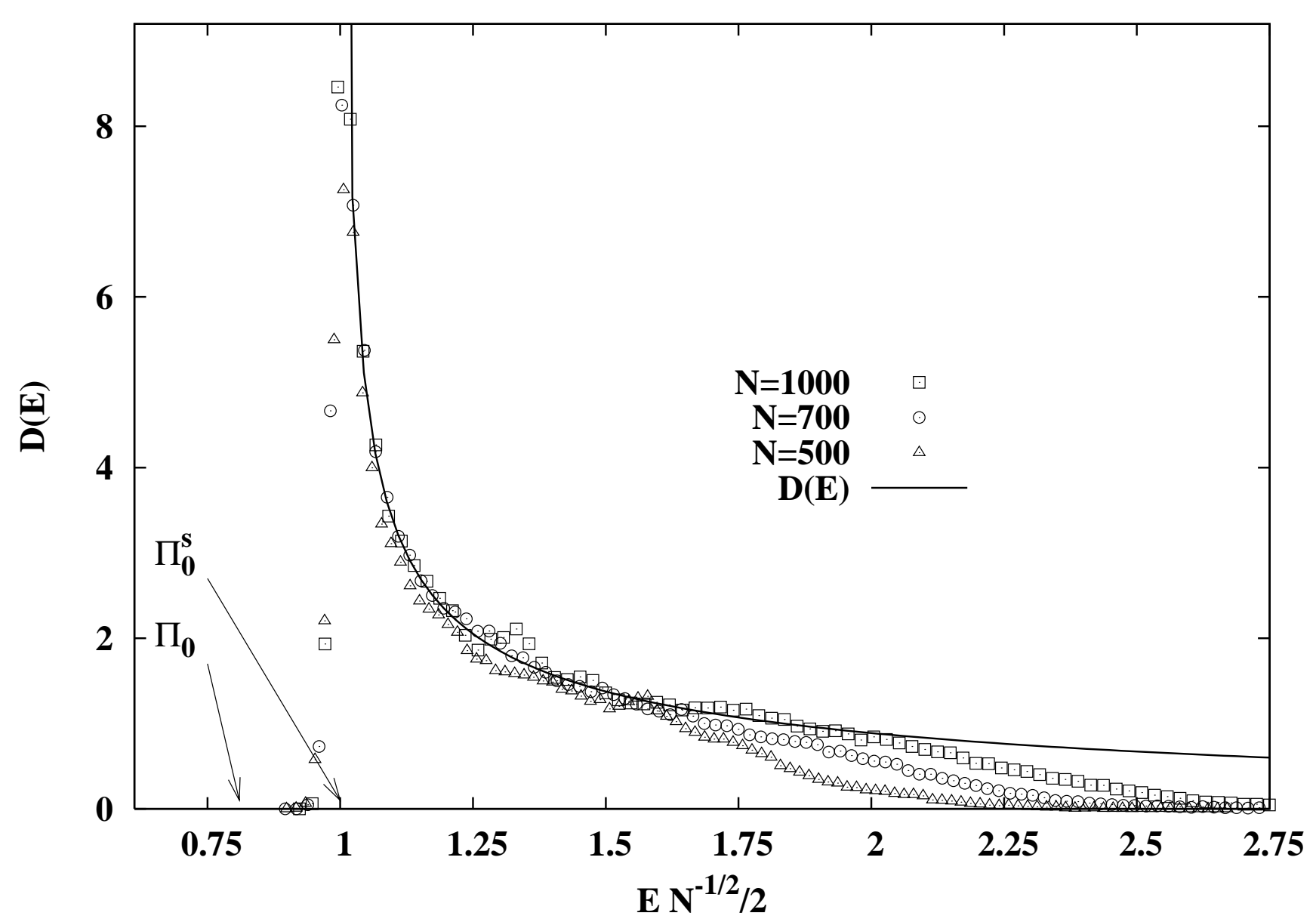

FIG. 5: Histogram of the energies of the stable states averaged over 1000 instances of the problem (symbols). The continuous line represent the analytical solution. (see eq. (13)

where $\Pi^{x}$ represents a matching. We as $\Pi^{a}$ and $\Pi^{b}$ the configurations of the stable marriage with lower energy $\left(\Pi_{0}^{s}\right)$ and the Global Optimum $\left(\Pi_{0}\right)$ respectively for the same set of preference lists.

Therefore, for a given set of preference lists of the players, we calculate the Global Optimum of the society, and determine the Stable Marriage State with lower energy (Optimum Stable State), then we calculate the distance between these two matchings. After averaging over 1000 instances we obtain that $D=0.53$ independently of $N$ (see figure (6). This result must be compared with the fact that the distance between two randomly taken matching goes as $1-1 / N$, see appendix $\nabla]$ that proves that there is a strong correlation between the two states. Approximately half of the couples are the same.

Moreover, we will like to know, from those couples that are different, how many of them and in which extend they improve their situation when the system moves from the Optimal Stable State to the Global Optimum. Again, averaging over 1000 instances we find that the $24 \%$ of the players improve his situations while the $29 \%$ get worse matches. However, the improvement of the formers is large enough to compensate the fact that more people get worst.

The results appear in figure 7 The data points marked by $\square$ represent the histogram (measured when the system is in the Global Optimum) of the energies of the men benefited in the transition from the Optimal Stable State to the Global Optimum. The points with $\bigcirc$ reflect the histogram of the energies of the same men but in the Optimum Stable State and those marked with $\triangle$ represent the histogram of all the men (not only those benefited) in the Optimum Stable State. As can be seen from the figure, the tail of the energy distribution of the men in the stable state disappears when the system moves to the Global Optimum. It means that the transition to the Global Optimum from the Optimum Stable State is achieved improving, mainly, the situation of the people matched worst in this state.

Analogously figure 8 reflects the opposite situation, in which a society goes from the Global Optimum to the Optimum Stable State. The points marked with $\square$ represent the histogram of the energy of all the men in the Optimum Stable State, while the other curves reflect the histogram of the energies of the men benefited with the 


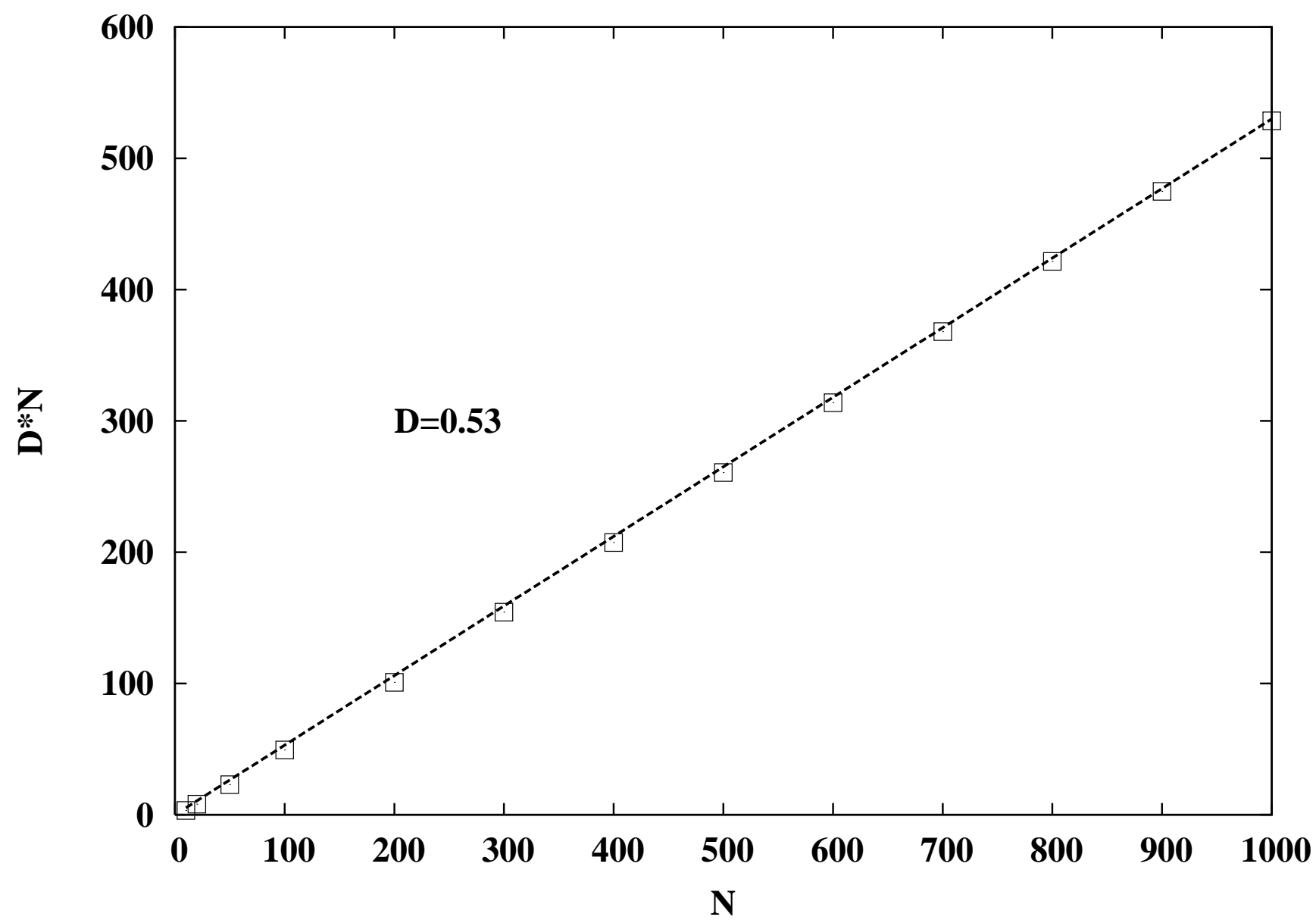

FIG. 6: Distance $N \cdot D\left(\Pi_{0}, \Pi_{0}^{s}\right)$ between the Global Minimum of the system and the Optimum Stable State. For each value of $N$ the points reflect averages over 1000 instances

transition (measured in the Optimum Stable State) $(\bigcirc)$ and the histogram of the energies of all the men in the Global Optimum $(\triangle)$. Again the tail of the distribution of the men in the Global Optimum contains those players that are benefited in the transition from the Global Optimum to the Optimum Stable State.

Then, the main conclusion to be drawn from figures 7 and 8 is that the players with higher energies would be the most motivated to make a transition from one state to the other (if they were informed about their presumed situation in the other state).

Moreover, it is interesting to find the number of unstable couples $N_{u c}$ in the Global Optimum. This is a better measure of the instability of the system and may give an idea of how many people will be tempted to act in self-interest if the society is in the Global Optimum.

Simple probabilistic arguments (see appendix VII) suggest that $N_{u c}$ growths linearly with $N$. This is, indeed, the case (see figure 9). Therefore, since $N_{u c}$ is a measure of how many couples are interested in acting for self-interesting the system is in the Global Optimum, considering that $N_{u c} \sim N$ and that the number of possible couples growths with $N^{2}$ the ratio of the couples to be forbidden to keep the system in the Global Optimum decreases as $1 / N$. This mean that the larger the society, the easy it is to keep it in the Global Optimum. While difficult to compare with real situations (where it has been never achieved the Global optimum) this result agrees with the standard notion that the larger the system the more stable it is. This is certainly also true in physical systems where the fluctuations decrease as $1 / \sqrt{N}$.

Finally, we ask, whether this perception of improvements felt by the player implied in unstable couples was real. In other words, how many of the people perceiving that may get benefits acting for self-interest will really find a better partner in the Nash state of lower energy. Studying by numerical simulations 100 systems of size $N=1000$ we concluded that the percentage of the people that have this feeling and will really improve his situation is nearly $80 \%$.

Summarizing, the results of this section suggest that, subject to evolutionary selection the self-fish attitude will 


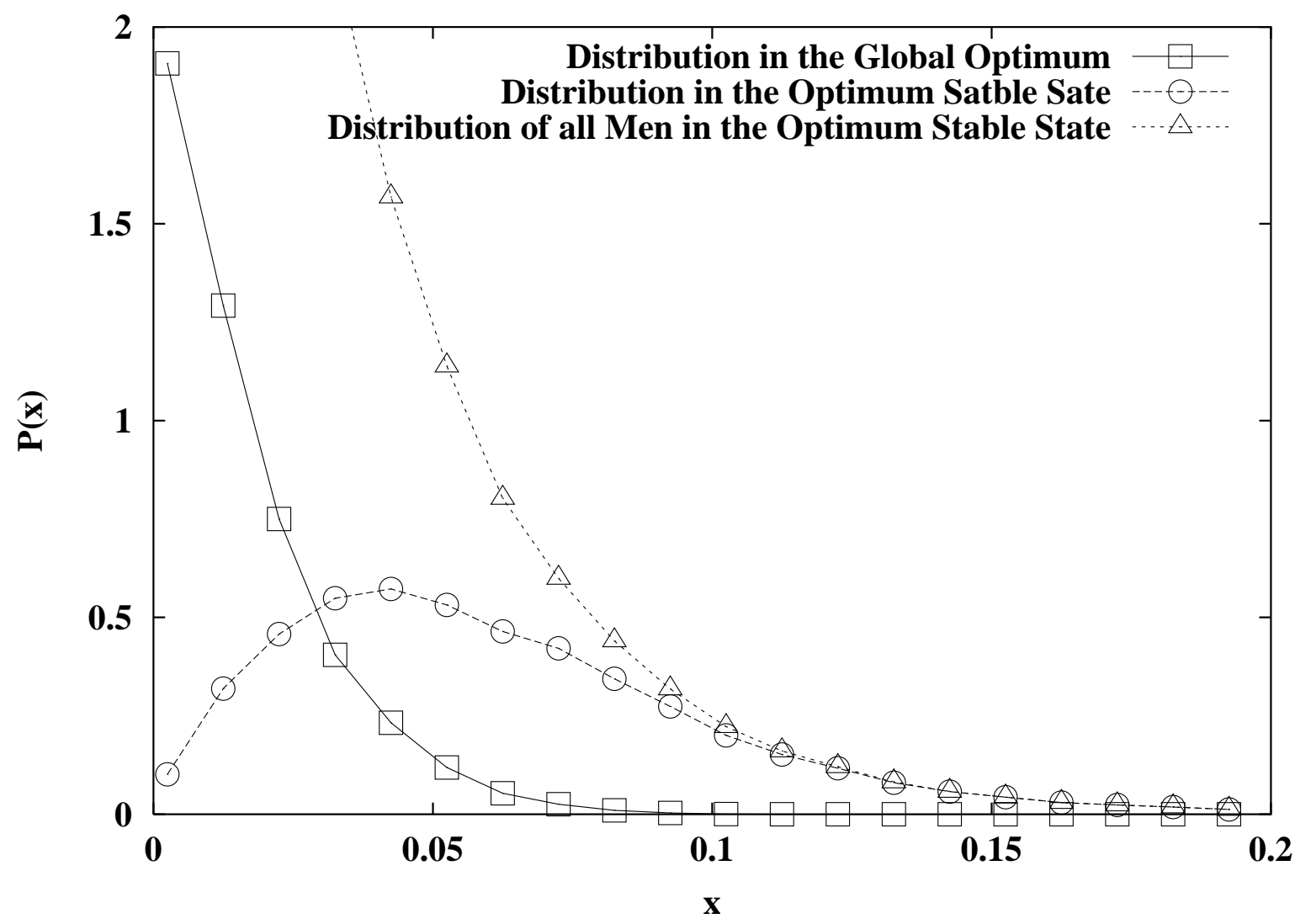

FIG. 7: Distribution of energies of the men that benefit from the transition to the Global Minimum for the Optimal Stable State. Every point represents an average over 100 instances of size $N=1000$

prevail over a Global Optimum in a competitive society. People that perceives that may improve their situation acting for his self-interest will really improve it taking the initiative and changing the partner.

\section{CONCLUSIONS}

We have studied the stable marriage problem from different point of views. We first proved that it is not easy to invent a simple microscopic dynamics for agents with limited information that lead to a Nash equilibrium state. However, we were able to characterize analytically one such dynamics that converges to a stationary state. From these results we proposed for the players a strategy that greatly improves their performance. We then focus our attention on the stable states of the marriage problem and derive a thermodynamical description of these states that agree very well with the results of Monte Carlo simulations. Finally, through large scale numerical comparison we compare the Global Optimum of the society with the stable marriage of lower energy. We showed that both state are strongly correlated and more interesting that the selffish attitude will indeed result in a benefit for most of the practitioners involved in unstable couples.

\section{APPENDIX A}

The master equation for $\rho(x, y)$

$$
\frac{\partial \rho}{\partial t}(x, y)=\int_{a, b=0}^{1}\left(P_{a, b \rightarrow x, y} \rho(a, b)-P_{x, y \rightarrow a, b} \rho(x, y)\right) d a d b
$$

though very intuitive has the terms $P_{a, b \rightarrow x, y}$ which are a little bizarre, and are not needed to do the calculation. The integration can be done directly without calculating explicitly this terms. It is suitable then to write the master 


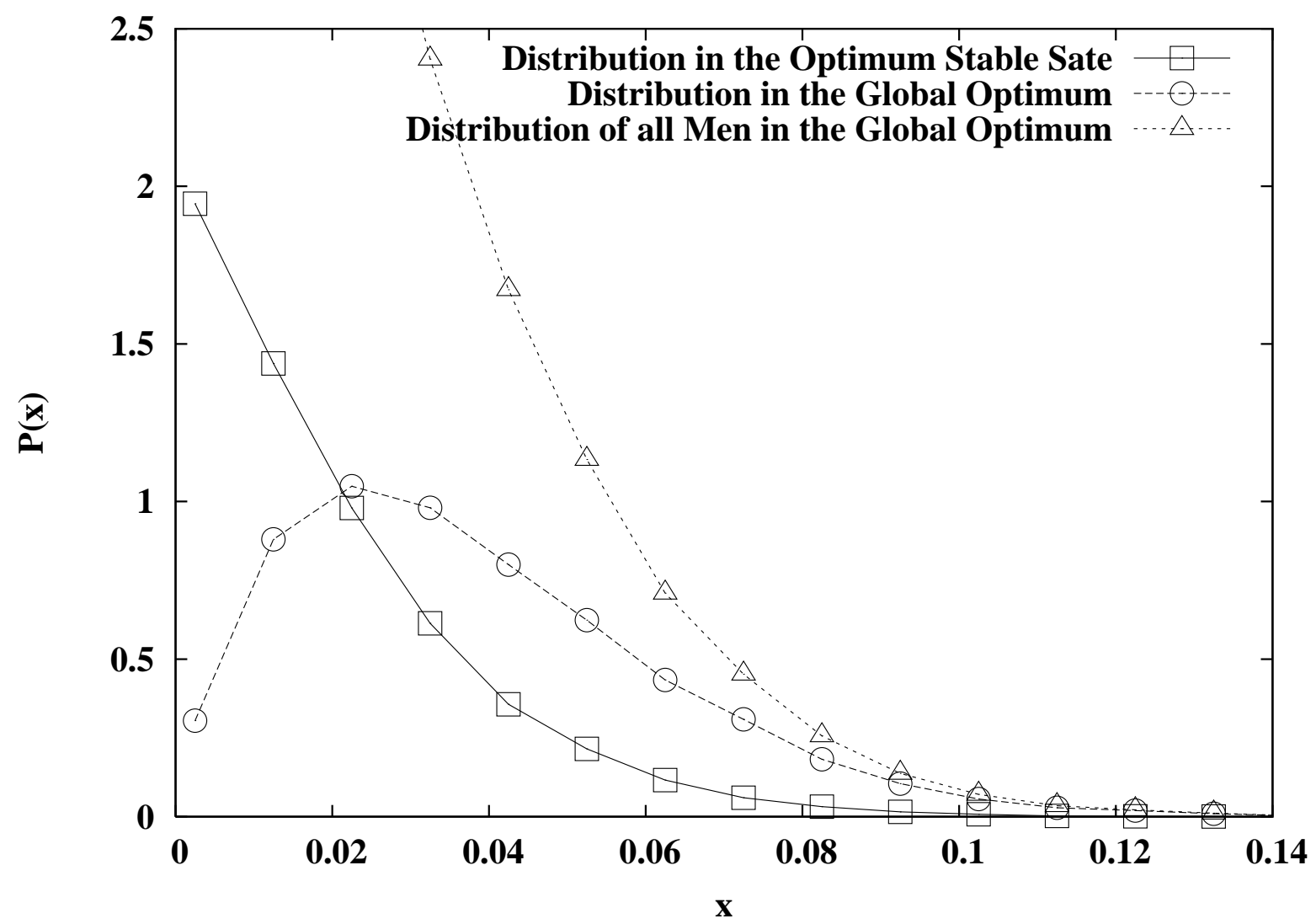

FIG. 8: Distribution of energies of the men that benefit from the transition to the Optimum Stable State from the Global Minimum. Every point represents an average over 100 instances of size $N=1000$

equation as a balance of probabilities like this:

$$
\frac{\partial \rho}{\partial t}\left(p_{0}\right)=W_{\rightarrow p_{0}}-W_{p_{0} \rightarrow}
$$

where $p_{0}=\left(x_{0}, y_{0}\right)$ is the energy vector of a couple and $W_{\rightarrow p_{0}}$ is the probability of having a situation in which a couple $p_{0}$ is created after an effective appointment, while $W_{p_{0}} \rightarrow$ is the probability of a situation in which such a couple finds itself involved in an effective appointment that breaks it apart. The position of the arrow stands for whether the effective appointment reaches or starts from a couple $p_{0}$.

In any appointment there are four couples to be considered: $p_{1}=\left(x_{1}, y_{1}\right)$ and $p_{2}=\left(x_{2}, y_{2}\right)$ which are the original couples, and $p_{1}^{\prime}=\left(x_{1}^{\prime}, y_{2}^{\prime}\right)$ and $p_{2}^{\prime}=\left(x_{2}^{\prime}, y_{1}^{\prime}\right)$ which are the possible new couples. Given that the primed values are taken randomly within $(0 \ldots 1)$, the probability density of a given point of the space of probabilities is $\rho\left(p_{1}, p_{2}, p_{1}^{\prime}, p_{2}^{\prime}\right)=$ $\rho\left(p_{1}\right) \rho\left(p_{2}\right)$, which is normalized. The situations in which a couple $p_{0}$ is obtained after an effective appointment happens both when $p_{1}^{\prime}=p_{0}$ and when $p_{2}^{\prime}=p_{0}$. While the situation in which a couple $p_{0}$ is destroyed takes place when $p_{1}=p_{0}$ and also when $p_{2}=p_{0}$. So the master equation can be written as:

$$
\frac{\partial \rho}{\partial t}\left(p_{0}\right)=W_{p_{1}^{\prime}=p_{0}}+W_{p_{2}^{\prime}=p_{0}}-W_{p_{1}=p_{0}}-W_{p_{2}=p_{0}}
$$

The probability of any given situation $A$ can be measured by the integration of a function $A\left(p_{1}, p_{2}, p_{1}^{\prime}, p_{2}^{\prime}\right)$ that is equal to 1 in the points of the probability space in which the situation occurs while is 0 in all other points. An appointment will be effective if $x_{1}^{\prime}<x_{1}$ and $y_{2}^{\prime}<y_{2}$. Then we can measure the situations $A_{p}$ in which $p=p_{0}$ and an effective appointment occurs through the function

$$
A_{p}=\theta\left(x_{1}-x_{1}^{\prime}\right) \theta\left(y_{2}-y_{2}^{\prime}\right) \delta\left(p-p_{0}\right)
$$

where $p$ can be either of the four couples involved in the appointment. 


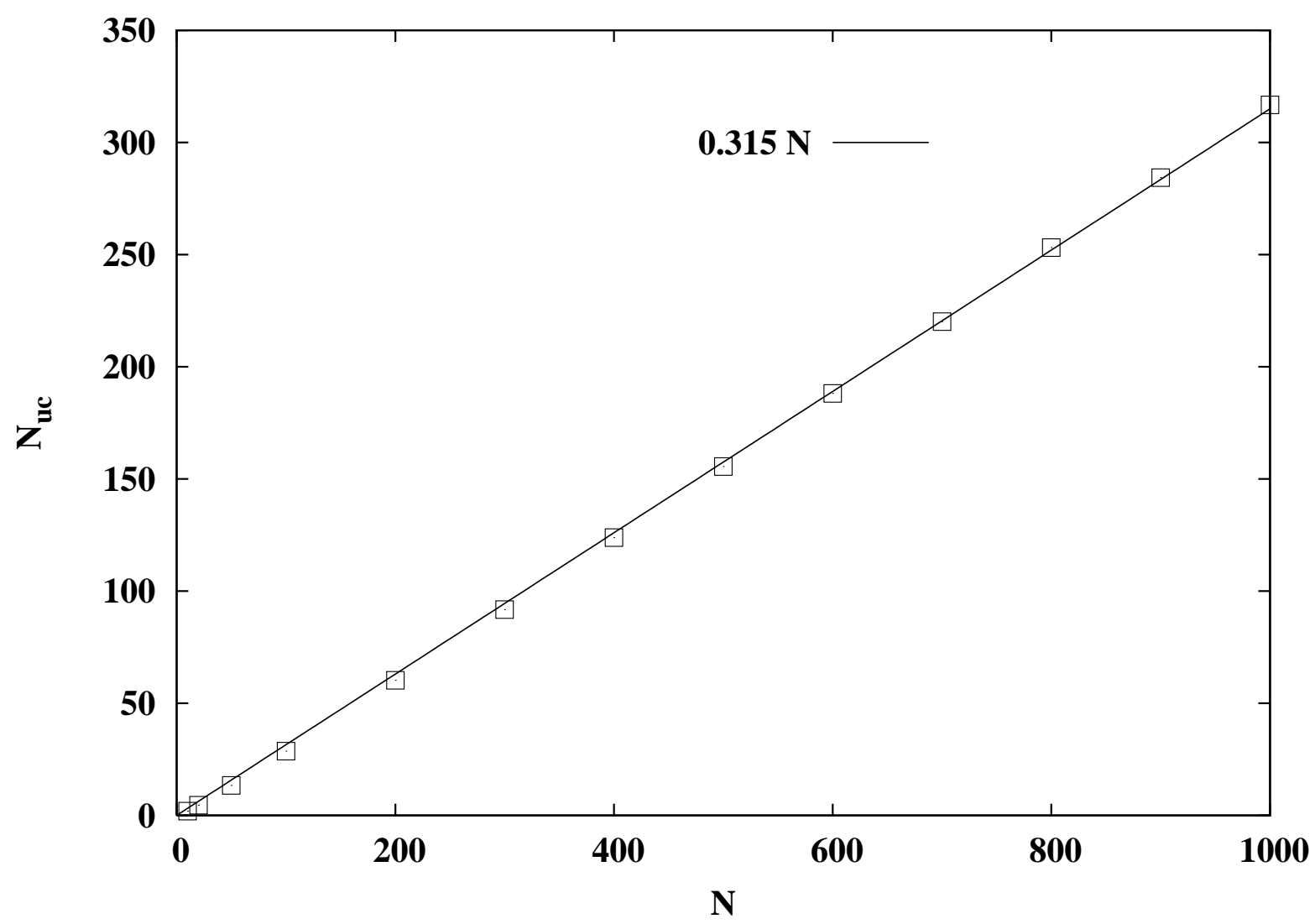

FIG. 9: Number of unstable couples $N_{u c}$ as a function of the system size $N$. The points reflect average values over 1000 instances

Any of the probability densities $W_{p}$ in equation (22) are given by:

$$
W_{p=p_{0}}=\int_{0}^{1} d p_{1} d p_{2} d p_{1}^{\prime} d p_{2}^{\prime} \rho\left(p_{1}\right) \rho\left(p_{2}\right) \theta\left(x_{1}-x_{1}^{\prime}\right) \theta\left(y_{2}-y_{2}^{\prime}\right) \delta\left(p-p_{0}\right)
$$

This integration is made straightforward. To show it let us take the case of $W_{p_{1}^{\prime}=p_{0}}$

$$
\begin{aligned}
W_{p_{1}^{\prime}=p_{0}} & =\int_{0}^{1} d p_{1} d p_{2} d p_{1}^{\prime} d p_{2}^{\prime} \rho\left(p_{1}\right) \rho\left(p_{2}\right) \theta\left(x_{1}-x_{1}^{\prime}\right) \theta\left(y_{2}-y_{2}^{\prime}\right) \delta\left(p_{1}^{\prime}-p_{0}\right) \\
& =\int_{0}^{1} d p_{1} d x_{1}^{\prime} d y_{1}^{\prime} \rho\left(p_{1}\right) \theta\left(x_{1}-x_{1}^{\prime}\right) \delta\left(x_{1}^{\prime}-x_{0}\right) \quad \int_{0}^{1} d p_{2} d x_{2}^{\prime} d y_{2}^{\prime} \rho\left(p_{2}\right) \theta\left(y_{2}-y_{2}^{\prime}\right) \delta\left(y_{2}^{\prime}-y_{0}\right) \\
& =\int_{0}^{1} d p_{1} \rho\left(p_{1}\right) \theta\left(x_{1}-x_{0}\right) \int_{0}^{1} d p_{2} \rho\left(p_{2}\right) \theta\left(y_{2}-y_{0}\right) \\
& =\int_{x_{0}}^{1} \int_{0}^{1} \rho(x, y) d x d y \quad \int_{0}^{1} \int_{y_{0}}^{1} \rho(x, y) d x d y
\end{aligned}
$$

Doing the same with the rest three terms of the master equation (22) we easily get:

$$
\begin{aligned}
\frac{\partial \rho}{\partial t}(x, y)= & \int_{0}^{1} \int_{x}^{1} \rho(a, b) d a d b \int_{y}^{1} \int_{0}^{1} \rho(a, b) d a d b+<x><y> \\
& -x<y>\rho(x, y)-y<x>\rho(x, y)
\end{aligned}
$$




\section{Appendix B}

There is a bijection between states of a system of size $N$ and the permutations of the numbers $(1,2 \ldots N)$ which results obvious if we consider that a given permutation $\Pi$, stands for the state with couples $\left\{\left(1, \Pi_{1}\right),\left(2, \Pi_{2}\right) . .\left(N, \Pi_{N}\right)\right\}$. Two random states are given by two random permutations $\Pi^{a}$ and $\Pi^{b}$.

Without lost of generality we can take one of this states fixed $\Pi^{a}=(1,2 \ldots N)$ while leaving all the randomness to the other. Now asking the distance is equivalent to ask how many of the numbers coincide with their position in the random permutation:

$$
\Pi=\begin{array}{llllll}
4 & 2 & 5 & 3 & 1 & 2 \\
1 & 2 & 3 & 4 & 5 & 6
\end{array}
$$

Lets say $P(k, N)$ is the probability that $k$ of $N$ numbers will coincide with their position in the random permutation. There are $\left(\begin{array}{l}k \\ N\end{array}\right)$ ways to pickup such $k$ numbers, and for each of these there are $(N-k)$ ! ways to order the rest $N-k$ numbers, of which a fraction $P(0, N-k) \simeq P(0, N)$ is such that no other number ends at its position, thus ensuring that only the first $k$ numbers do. Then we have a relation between $P(k, N)$ and $P(0, N)$ :

$$
\begin{aligned}
P(k) & =\frac{\left(\begin{array}{l}
k \\
N
\end{array}\right)(N-k) ! P(0)}{N !} \\
& =\frac{P(0)}{k !}
\end{aligned}
$$

$P(0)$ can be calculated by the inclusion exclusion principle as:

$$
\begin{aligned}
P(0) & =\sum_{q=0}^{q=N} \frac{(-1)^{q+1}}{q !} \\
& \simeq e^{-1}
\end{aligned}
$$

Then the mean value of $k$ is:

$$
\begin{aligned}
\bar{k} & =\sum_{k=0}^{k=N} k P(k)=\frac{1}{e} \sum_{k=0}^{k=N} \frac{k}{k !}=\frac{1}{e} \sum_{k=0}^{k=N-1} \frac{1}{k !}=\frac{1}{e} e \\
& =1
\end{aligned}
$$

and the distance between random states is in average:

$$
\begin{aligned}
D\left(\Pi^{a}, \Pi^{b}\right) & =\frac{1}{N} \sum_{i=1}^{N}\left(1-\delta_{\Pi_{i}^{a}, \Pi_{i}^{b}}\right) \\
& =\frac{N}{N}-\frac{\sum_{i=1}^{N} \delta_{\Pi_{i}^{a}, \Pi_{i}^{b}}}{N} \\
& =1-\frac{\bar{k}}{N} \\
& =1-\frac{1}{N} \sim 1
\end{aligned}
$$

\section{Appendix C}

A mean field approximation to the number of unstable couples $N_{u c}$ can be work out considering that a man with energy $x$ and a woman with energy $y$ form an unstable couple with probability $x y$. Then, in average any two persons $m$ and $w$ will form an unstable couple with probability: 


$$
\begin{aligned}
P_{h, m} & =\int_{0}^{1} \int_{0}^{1} P(x) P(y) x y d x d y \\
& =\int_{0}^{1} P(x) x d x \int_{0}^{1} P(y) y d y
\end{aligned}
$$

where $P(x)$ is the probability density of the energies of men and women in the Global Optimum. The integrals are nothing but the averages values of the energies of men and women, which are related to the the total energy of men $X$ and women $Y$ as $x=X / N$ and $y=Y / N$. In the fundamental state $X=Y=\frac{E^{0}}{2}=0.81 \sqrt{N}$ then

$$
\begin{aligned}
P_{h, m} & =\frac{X}{N} \frac{Y}{N}=\left(\frac{E^{0}}{2 N}\right)^{2} \\
& =\frac{0.654}{N}
\end{aligned}
$$

$P_{m, w}$ is the probability that a generic couple $(m, w)$ is an unstable couple. Then, any man $m$ belongs to $N P(m, w)$ unstable couples, and, as there are $N$ men, the total number of unstable couples will be:

$$
\begin{aligned}
N_{u c} & =N^{2} P_{m, w} \\
& =0.654 N \\
& \propto N
\end{aligned}
$$

\section{ACKNOWLEDGMENTS}

We thank C. Trallero-Giner for useful discussions and comments. We also acknowledge the support of the NET-61 from the ICTP.

[1] D. Gusfield and R.W. Irving, The stable marriage problem, MIT Press, Cambridge, MA, 1989

[2] R. Gibons, A Primer in Game Theory, Harvester Wheatsheaf, 1992

[3] M. Mezard and G. Parisi, J. Physique Lett 46 (1985) L771

[4] M.-J. Omero, M. Dzierzawa, M. Marsili and Y.-C. Zhang, J. Phys. I France 7 (1997) 1723

[5] M. Dzierzawa and M.-J. Omero, Physica A 287, (2000) 321

[6] P. Laureti and Y.-C. Zhang, Physica A 324,(2003) 49

[7] G. Caldarelli and A. Capocci, cond-mat/0008337

[8] K. Huang, Introduction to Statistical Physics, London, Taylor and Francis, 2001 